\title{
Dinâmica populacional de ácaros fitófagos (Acari, Eriophyidae, Tenuipalpidae) em seis clones de seringueira no sul do Estado de Mato Grosso
}

\author{
Rodrigo Damasco Daud ${ }^{1,2} \&$ Reinaldo José Fazzio Feres ${ }^{2}$
}

\begin{abstract}
${ }^{1}$ Programa de Pós Graduação em Biologia Animal. Bolsista Capes. rodrigodaud@yahoo.com.br
${ }^{2}$ Departamento de Zoologia e Botânica, Universidade Estadual Paulista - UNESP, campus de São José do Rio Preto, SP. Rua Cristóvão Colombo, 2265, Jardim Nazareth, 15054-000 São José do Rio Preto-SP. reinaldo@ibilce.unesp.br
\end{abstract}

\begin{abstract}
Population dynamics of phytophagous mites (Acari, Eriophyidae, Tenuipalpidae) on six rubber trees clones in southern Mato Grosso State. The population dynamics of Calacarus heveae Feres, 1992, Phyllocoptruta seringueirae Feres, 1998 (Eriophyidae) and Tenuipalpus heveae Baker, 1945 (Tenuipalpidae) was studied on six rubber tree clones (Hevea brasiliensis Muell. Arg.) in south of Mato Grosso State, Brazil, in order to verify the seasonality and the possible differences on the infestation levels of these mites. The samplings were made from March 2004 to March 2005, with an average interval of fourteen days between each sampling. In each sampling, 100 leaflets were taken from each clone crop, and the total number of mites found in both leaf surfaces was registered. The infestation levels of mites differed among the clones. The clone RRIM 600 showed the highest number of T. heveae, while PB 235 and PB 260 had the highest abundance of $P$. seringueirae and $C$. heveae, respectively. On the other hand, this latter clone had the smallest infestation of $T$. heveae and $P$. seringueirae, while in PB 235 the lowest population of $C$. heveae. The critical period of mite infestation was observed in the first semester, although, there was variation in the duration of phytophagous occurrence and the infestation peak on six rubber trees clones studied.
\end{abstract}

KEYWORDS. Hevea brasiliensis; infestation; pest mites; seasonality.

RESUMO. Dinâmica populacional de ácaros fitófagos (Acari, Eriophyidae, Tenuipalpidae) em seis clones de seringueira no sul do Estado de Mato Grosso. Foi estudada a dinâmica populacional de Calacarus heveae Feres, 1992, Phyllocoptruta seringueirae Feres, 1998 (Eriophyidae) e Tenuipalpus heveae Baker, 1945 (Tenuipalpidae) em seis clones de seringueira (Hevea brasiliensis Muell. Arg.), com o intuito de conhecer a sazonalidade e as possíveis diferenças nos níveis de infestação dessas espécies sobre os diferentes clones, em seringal localizado no sul do Estado de Mato Grosso. Para isso, no período de março de 2004 a março de 2005, foram realizadas coletas num intervalo médio de catorze dias em seis parcelas clonais. Em cada amostragem, foram analisados 100 folíolos de cada parcela, registrando-se o número total de ácaros encontrados em ambas as faces. Os níveis de infestação das espécies variaram entre os diferentes clones. O clone RRIM 600 apresentou maior abundância de T. heveae, enquanto que PB 235 e PB 260 de P. seringueira e C. heveae, respectivamente. Por outro lado, esse último clone apresentou menor número de indivíduos de $T$. heveae e $P$. seringueirae, e PB 235 de $C$. heveae. O primeiro semestre foi o período crítico de infestação, porém a duração da ocorrência e o pico de abundância dos fitófagos variaram nos diferentes clones.

PALAVRAS-CHAVE. Hevea brasiliensis; infestação; pragas; sazonalidade.

A seringueira (Hevea brasiliensis Muell. Arg., Euphorbiaceae), planta nativa da Região Amazônica, é a maior fonte de borracha natural (Gonçalves et al. 2001). Devido à sua importância econômica, essa planta foi introduzida em várias regiões do Brasil na forma de monocultura.

Esse sistema de plantio, associado aos fatores edafoclimáticos desses locais, propiciaram condições para o surgimento de espécies-praga. O Estado de Mato Grosso é considerando o terceiro maior produtor de borracha, responsável por $16,2 \%$ da produção nacional, ficando atrás do Estado de São Paulo (60,9\%) e Bahia (20,5\%) (IAC 2006).

Calacarus heveae Feres, 1992 (Acari, Eriophyidae) tem sido considerado o principal ácaro-praga da seringueira nas regiões sudeste e centro-oeste do Brasil (Feres 2000; Ferla \& Moraes 2002; Gallo et al. 2002). Vieira et al. (2000) afirmaram que dependendo de diversos fatores ambientais e intrínsecos das plantas, o ataque de $C$. heveae pode ocasionar dois tipos de sintomas nos folíolos: (1) amarelecimento progressivo dos folíolos intercalados por áreas verdes, sendo que as manchas amareladas, com o passar do tempo, podem tornar-se avermelhadas, e (2) pontuações amarelas, com ou sem áreas necróticas centrais. Grandes infestações dessa espécie podem provocar níveis de desfolha acima de $75 \%$ e ocasionar, segundo alguns produtores, perdas de até $30 \%$ na produção de látex, matéria-prima na fabricação da borracha (Vieira \& Gomes 1999; Feres 2000). Ferla \& Moraes (2003a) estudaram o ciclo biológico dessa espécie no clone PB 260 e registraram um período de cerca de nove dias da fase de ovo a adulto e fecundidade média de 16,2 ovos/fêmea. O controle dessa espécie tem sido realizado somente com defensivos agrícolas, sendo o bromopropilato, o mais eficiente desses produtos (Ferla \& Moraes 2003b; Vieira \& Gomes 1999, 2001).

Outros ácaros fitófagos da seringueira que têm merecido atenção são Tenuipalpus heveae Baker, 1945 (Acari, 
Tenuipalpidae) e Phyllocoptruta seringueirae Feres, 1998 (Acari, Eriophyidae). Tenuipalpus heveae, diferentemente de C. heveae, ocorre preponderantemente na face abaxial, e em grandes infestações pode provocar sintomas de ferrugem nos folíolos e intensa desfolha nos seringais (Pontier et al. 2000). Além disso, alguns estudos revelaram a sua resistência a alguns defensivos agrícolas (Feres et al. 2002; Ferla \& Moraes 2003b). Tenuipalpus heveae tem um período de desenvolvimento relativamente longo, cerca de 23,5 dias de ovo a adulto e deposita seus ovos principalmente ao longo das margens da nervura central e em outros lugares protegidos do folíolo (Pontier et al. 2000). P. seringueirae tem sido relatado com freqüência em seringais paulistas e mato-grossenses, porém, é normalmente registrado em menor abundância (Feres et al. 2002; Ferla \& Moraes 2002; Bellini et al. 2005) e, aparentemente, não provoca danos significativos às plantas.

O estudo da dinâmica populacional de espécies-praga pode favorecer a realização de práticas eficientes de manejo, com a determinação do período de ocorrência das infestações, do pico populacional e da época em que ocorre o reaparecimento das espécies no campo. A flutuação populacional de ácaros em monoculturas de seringueira foi estudada por alguns autores que realizaram amostragens mensais dos ácaros associados ao clone RRIM 600 (Bellini et al. 2005; Hernandes \& Feres 2006; Vis et al. 2006). O objetivo deste trabalho foi conhecer a sazonalidade e as possíveis diferenças nos níveis de infestação de $C$. heveae, $P$. seringueirae e $T$. heveae em seis diferentes clones, em seringal localizado no sul do Estado de Mato Grosso.

\section{MATERIALE MÉTODOS}

Áreas de estudo. Pertencem à Fazenda Plantações E. Michelin Ltda. - PEM $\left(17^{\circ} 23^{\prime} \mathrm{S}, 54^{\circ} 45^{\prime} \mathrm{W}\right)$, município de Itiquira, MT; propriedade com 8.484,52 ha de área cultivada com mais de 100 clones de seringueira. Foram utilizadas as áreas denominadas "CCGE 1 e CCGE 2", cujas plantas tinham cerca de 18 anos de idade, isentas de aplicação de agrotóxicos desde dezembro de 2002. Foram estudados os clones GT 1, PB 217, PB 235, PB 260, PR 255 e RRIM 600, cultivados em parcelas de cerca de 0,42 ha cada.

Amostragens. As coletas foram realizadas num intervalo médio de 14 dias, da segunda quinzena de março de 2004 ao início de março de 2005. A amostragem foi limitada ao interior do cultivo de cada clone para se evitar o efeito de borda. Em cada coleta e em cada clone, foram tomadas aleatoriamente, com auxílio de um podão com cabo telescópico, 10 folhas ao redor da copa de 10 diferentes plantas até uma altura de $7 \mathrm{~m}$, totalizando 100 folhas por parcela clonal. As folhas coletadas foram acondicionadas em sacos de papel, separados por clone, e armazenadas em caixas isotérmicas de poliestireno. Esse material foi enviado por via aérea, no mesmo dia da coleta, para o laboratório de Acarologia da UNESP, São José do Rio Preto, SP, sendo recebido num período médio de 48 horas. Em laboratório, o material foi armazenado a $10^{\circ} \mathrm{C}$ por no máximo 10 dias, até ser totalmente analisado. Para o estudo da dinâmica populacional, um folíolo de cada folha amostrada foi analisado sob microscópio estereoscópico, sendo registrado o número total de ácaros encontrados em ambas as faces. Cerca de 15 exemplares de cada espécie de ácaro, selecionados ao acaso, foram montados em lâminas de microscopia com o meio de Hoyer (Flechtmann 1975) e depositados na coleção de Acari (DZSJRP) - http://www.splink.cria.org.br, do Departamento de Zoologia e Botânica, Universidade Estadual Paulista (UNESP), São José do Rio Preto, SP, como material testemunho.

Os parâmetros climáticos foram obtidos através da estação meteorológica da PEM, localizada cerca de $2.160 \mathrm{~m}$ de distancia da área de estudo. Os parâmetros analisados, considerandose os cinco dias que antecederam as coletas, foram: temperatura média, umidade relativa média do ar, tempo médio de insolação diária e pluviosidade total.

Análises estatísticas. O nível de infestação das três espécies estudadas nos diferentes clones foi comparada através do teste G. Correções de Bonferroni $(\alpha / \mathrm{n})$ foram usadas para comparações pareadas. Esse teste também foi aplicado para comparar a incidência de chuvas entre dois períodos que antecederam as infestações de $C$. heveae: (1) novembro de 2003 a janeiro de 2004 e (2) novembro de 2004 a janeiro de 2005. A umidade relativa média do ar registrada nesses dois períodos foi comparada pelo teste não-paramétrico de MannWhitney (Zar 1999).

Não foram considerados nas análises os dados referentes ao período de senescência das folhas.

\section{RESULTADOS}

$\mathrm{O}$ ácaro fitófago mais abundante registrado foi $T$. heveae com 180.662 indivíduos coletados, seguido pelos eriofí́deos P. seringueirae e C. heveae, com 144.353 e 9.915 espécimens, respectivamente.

Os níveis de infestação dos ácaros fitófagos diferiram entre os clones de seringueira. No clone RRIM 600, foi registrada a maior infestação de $T$. heveae $(\mathrm{G}=42.670,2, \mathrm{gl}=5, P<0,001)$ (Fig 1A). P. seringueirae foi mais abundante em PB $235(\mathrm{G}=$ 49.977,7, $\mathrm{gl}=5, P<0,001$ ), enquanto que $C$. heveae em PB 260 $(\mathrm{G}=10.177,5, \mathrm{gl}=5, P<0,001)$ (Fig. 1A e B). Por outro lado, nesse último clone, foram registradas as menores infestações de $T$. heveae e P. seringueirae, e em PB 235, a de $C$. heveae. Embora em número inferior em relação aos clones mais infestados, foi também observado grande número de indivíduos de $P$. seringueirae e $T$. heveae em PR 255, e de $C$. heveae em RRIM 600. Os clones GT 1 e PB 117 apresentaram níveis intermediários de abundância dos três fitófagos estudados (Fig. 1A e B).

A maior abundância das três espécies foi observada nas coletas realizadas no primeiro semestre do ano, antes do período de senescência natural das folhas, que variou em cada clone de seringueira (Fig. 2A-F). O período de ocorrência mais longo de T. heveae foi observado no clone GT 1, cuja infestação ocorreu da segunda quinzena de março até a primeira quinzena de julho de 2004 (Fig. 2A), enquanto que o de menor duração foi observado em PR 255 e RRIM 600, com infestação 

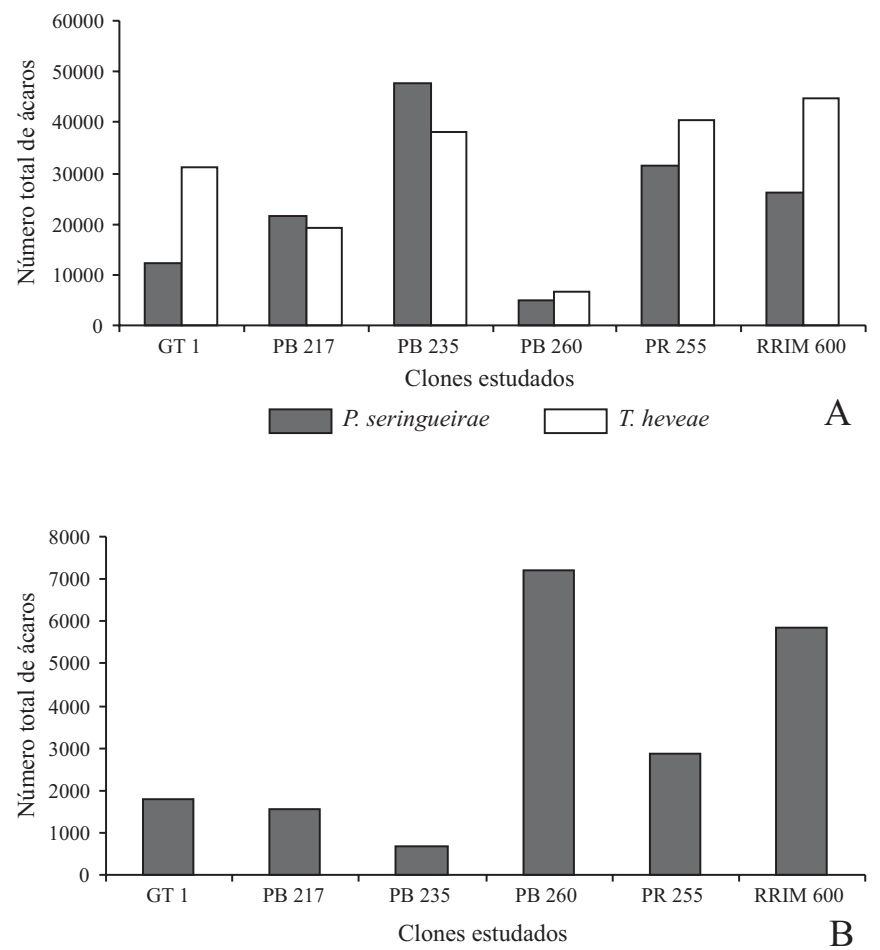

Fig. 1. Abundância total de ácaros nos seis cultivos de clones de seringueira, na Fazenda Plantações E. Michelin Ltda., Itiquira, MT, no período de março de 2004 a março de 2005. (A) Tenuipalpus heveae e Phyllocoptruta seringueirae, e (B) Calacarus heveae.

perdurando de março até 31 de maio de 2004 (Fig. 2E e F). Nos demais, a infestação dessa espécie ocorreu até junho de 2004 (Fig. 2B-D). Após a senescência das folhas, no período seco do ano (Fig. 3), a população de T. heveae ocorreu em número reduzido em todos os clones estudados, voltando a se elevar somente a partir de dezembro de 2004 nos clones PB 217, 260, PR 255 e RRIM 600, sendo nesse último em maior número $(\mathrm{G}=$ $3.017,2, \mathrm{gl}=4, P<0,001)$, e nos demais, a partir de janeiro de 2005 (Fig. 2A-F).

O pico populacional de $T$. heveae variou entre os clones de seringueira. O pico de abundância nos clones PR 255 e GT 1 foi registrado na primeira e segunda quinzena de abril de 2004, respectivamente, período com altos índices pluviométricos e temperatura elevada (Fig. 3). Em PB 217, o pico populacional de $T$. heveae ocorreu na segunda quinzena de fevereiro, enquanto que em PB 235, 260 e RRIM 600 na primeira quinzena de março de 2005 , período esse, antecedido por meses chuvosos e de umidade relativa e temperatura elevadas (Figs. 2A-E e 3).

O período de ocorrência mais longo de $P$. seringueirae foi observado nos clones PB 217, 235, 260 e RRIM 600, com a infestação ocorrendo de março até o final de junho de 2004. Nos demais, a população já estava reduzida nesse período, permanecendo assim até o início de janeiro de 2005 (Fig. 2AF). No clone PB 235, houve um aumento discreto de $P$. seringueirae após a senescência das folhas, na primeira quinzena de setembro de 2004, entretanto, após essa data,
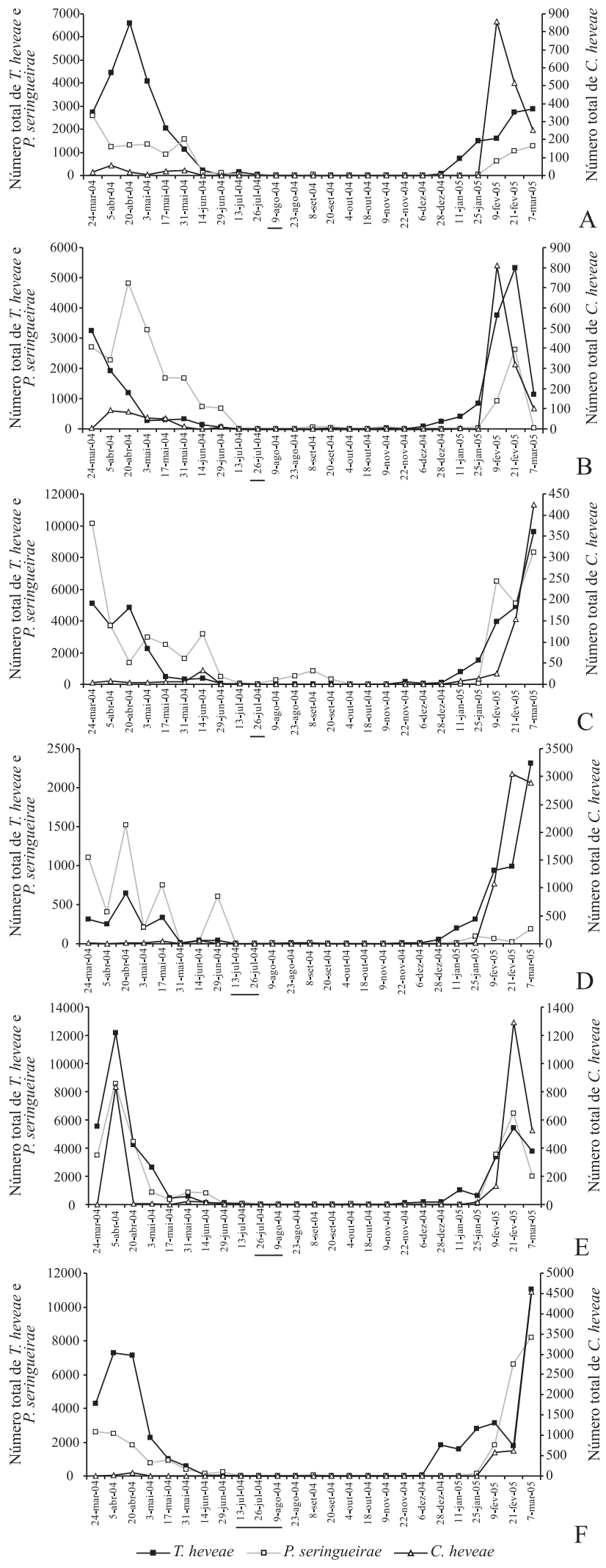

Fig. 2. Dinâmica populacional das três espécies de ácaros fitófagos estudadas, nos diferentes cultivos de clones de seringueira, Fazenda Plantações E. Michelin, Ltda., Itiquira, MT, no período de 24 de março de 2004 a 07 de março de 2005. Clones: (A) GT 1, (B) PB 217, (C) PB 235, (D) PB 260, (E) PR 255, e (F) RRIM 600. Barra: Período natural de senescência. 


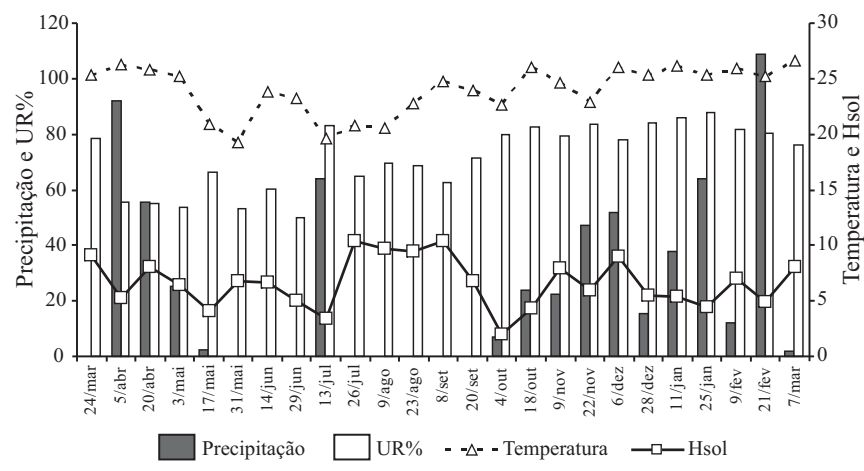

Fig. 3. Precipitação total $(\mathrm{mm})$, umidade relativa (UR\%), Temperatura $\left({ }^{\circ} \mathrm{C}\right)$ e horas de sol média (Hsol) nos cinco dias que antecederam as coletas, realizadas na Fazenda Plantações E. Michelin Ltda., Itiquira, MT, entre 24 de março de 2004 a 07 de março de 2005.

houve queda populacional (Fig. 2C). Em todos os clones, a população de $P$. seringueirae voltou a crescer a partir de fevereiro de 2005, em plena estação chuvosa (Fig. 3), atingindo maior abundância no clone PB $235(\mathrm{G}=12.629,1, \mathrm{gl}=5, P<$ 0,001) (Fig. 2A-F). A ocorrência de P. seringueirae foi sincrônica com $T$. heveae ao longo do ano, embora os picos populacionais e a duração do período de infestação dessas espécies tenham diferido entre os clones estudados.

Similarmente a $T$. heveae, registraram-se diferentes picos populacionais de $P$. seringueirae nos seis clones. Nos clones GT 1 e PB 235, o pico de abundância ocorreu em março, enquanto que em PB 217, 260 e PR 255 em abril de 2004. Já em RRIM 600 foi registrado no inicio de março de 2005 (2A-F).

Calacarus heveae foi pouco abundante na maioria dos clones no primeiro semestre de 2004, exceto em PR 255 no início de abril (Fig. 2E). Esse fitófago ocorreu de março a maio em GT 1, PB 217 e RRIM 600, e até junho em PB 235, 260 e PR 255. Por outro lado, após janeiro de 2005, C. heveae foi abundante e atingiu pico populacional em fevereiro de 2005 nos clones GT 1, PB 217, 260 e PR 255 e em março (final da estação chuvosa e inicio da seca, fig. 3) nos clones PB 235 e RRIM 600 (Fig. 2A-F).

A infestação de $C$. heveae em 2005 foi antecedida por período com chuvas mais intensas $(\mathrm{G}=50,44, \mathrm{gl}=1, \mathrm{p}<0,001)$ e com maior umidade relativa do ar $(Z=4,7, \mathrm{p}<0,001)$ (Período 2) em relação à mesma época em 2004 (Período 1) (Fig. 4A e B).

\section{DISCUSSÃO}

Calacarus heveae tem sido registrado como o ácaro mais abundante em seringueira no Estado de São Paulo (Feres 2000; Gallo et al. 2002; Bellini et al. 2005; Demite \& Feres 2005; Hernandes \& Feres 2006; Vis et al. 2006). Infestações de $T$. heveae, principalmente em fevereiro, já foram relatadas por Feres (2000) e Pontier et al. (2000) em seringais de Barretos, SP e de Pontes e Lacerda, MS, respectivamente. Feres et al. (2002) registraram infestações dessa espécie em seringal de Taquaritinga, SP, onde havia aplicação periódica de pesticidas. Problemas de infestações de T. heveae também foram observados por J.F.C. Benesi (com. pess.) em seringais de Goianésia, GO. Apesar do registro de sua grande abundância em alguns seringais (Feres et al. 2002; Ferla \& Moraes 2002; Bellini et al. 2005), infestações de P. seringueirae, nos níveis registrados neste trabalho, não haviam sido relatadas até o momento. Além da grande abundância, o período de ocorrência dessas duas espécies foi semelhante. Como ambas habitam preferencialmente a face abaxial dos folíolos, não foi possível a caracterização dos danos causados nos folíolos por cada uma dessas duas espécies.

As maiores infestações de $C$. heveae foram registradas nos clones PB 260 e RRIM 600 (o segundo mais infestado) e a menor em PB 235. Por outro lado, Furquim (1999) apud Tanzini (1999) observou maiores incidências desse ácaro em PB 235 e menores em RRIM 600 e GT 1, num seringal localizado no município de Jaboticabal, SP. Segundo Gallo et al. (2002), as condições climáticas e edáficas, a idade e as condições fisiológicas da planta, além de vários outros fatores, podem influenciar na manifestação da resistência de uma espécie vegetal num determinado período, o que pode explicar o diferente resultado encontrado neste trabalho.

No clone GT 1 foi registrada a maior duração no período de infestação de $T$. heveae, sendo que sua população diminuiu somente a partir de julho. Porém, o reaparecimento dessa espécie em campo foi mais rápido e mais intenso em RRIM 600 , pois foi nesse clone que a população voltou a aumentar a partir de dezembro. O período de infestação de P. seringueira foi maior em PB 235, com a infestação ocorrendo de março até junho de 2004. A recuperação populacional dessa espécie em PB 235 foi mais rápida em relação aos demais, pois $P$. seringueirae foi mais abundante nesse clone logo no início
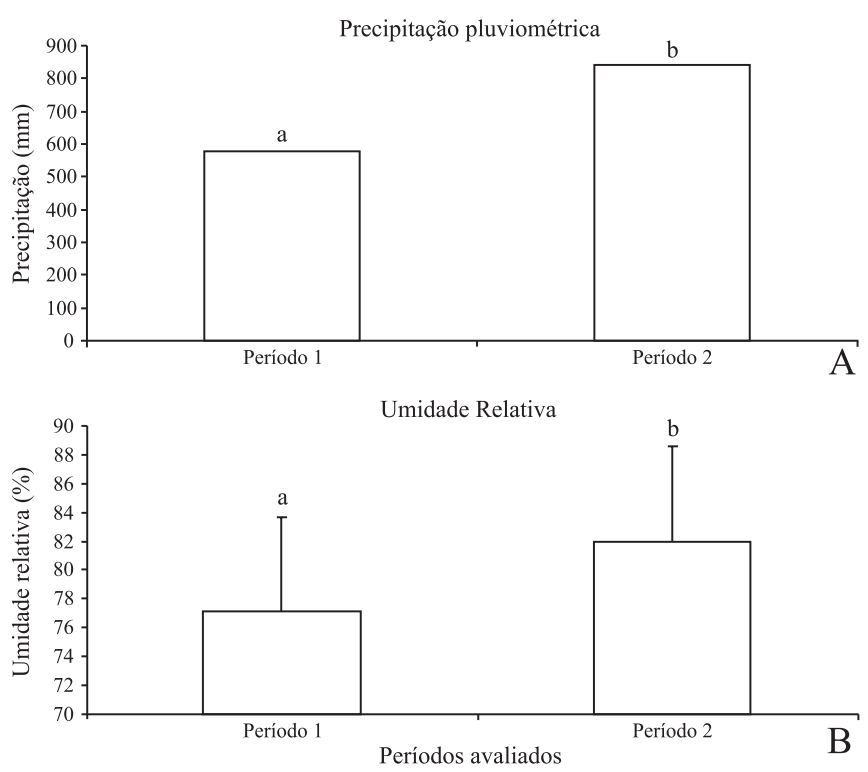

Fig. 4. Precipitação total (A) e umidade relativa média (B) em plena estação chuvosa, de novembro de 2003 a janeiro de 2004 (Período 1) e de novembro de 2004 a janeiro de 2005 (Período 2), na Fazenda Plantações E. Michelin Ltda, Itiquira, MT. Diferentes letras entre as barras significam diferenças estatísticas ao nível de 5\% de probabilidade. 
do período de re-infestação (fevereiro de 2005). As maiores infestações registradas e a intensidade do ressurgimento das populações são indicativas de que os clones RRIM 600 e PB 235 podem apresentar menor resistência ao ataque de $T$. heveae e $P$. seringueirae, respectivamente.

O primeiro semestre foi o período crítico de infestação das três espécies nos seringais de Itiquira, MT. Outros autores também observaram esse padrão de ocorrência em seringais de outras localidades. Vieira \& Gomes (1999) observaram que o primeiro semestre foi o período mais crítico de infestação de C. heveae em seringal de Reginópolis, SP. Feres et al. (2002), Bellini et al. (2003) e Hernandes \& Feres (2006) registraram maior abundância dessas três espécies nos meses correspondentes ao término da estação chuvosa e inicio da estação seca na região noroeste do Estado de São Paulo, que corresponde ao período de fevereiro a maio. Já Vis et al. (2006) verificaram grande infestação de $C$. heveae entre abril e maio em seringal de Piracicaba, SP. Portanto, se adoção de medidas de controle forem consideradas necessárias, deverão ser feitas entre janeiro e fevereiro, período em que as populações dos ácaros citados encontram-se em maior expansão.

A infestação de C. heveae em 2005 foi antecedida por um período com maior índice pluviométrico e, consequientemente, com maior umidade relativa do ar, do que a mesma época em 2004. Esses fatores ambientais podem ter propiciado melhores condições para o desenvolvimento desse ácaro. Ferla \& Moraes (2003a) obtiveram êxito na criação de $C$. heveae somente com o aumento da umidade relativa nas arenas de criação, e Hernandes \& Feres (2006) registraram maior incidência dessa espécie em períodos que foram antecedidos por meses com maior regime de chuvas.

Agradecimentos. À empresa Plantações E. Michelin Ltda., Itiquira, MT, pelo financiamento do estudo. Aos Engenheiros Agrônomos Fernando S. Fonseca e Cássio H.J. Scomparin, pelas informações concedidas sobre os cultivos estudados e aos técnicos Julio C. de Andrade e Maria de L. S. Siqueira da mesma empresa, pela realização das amostragens.

\section{REFERÊNCIAS}

Bellini, M. R.; G. J. de Moraes \& R. J. F. Feres. 2005. Ácaros (Acari) de dois sistemas de cultivo da seringueira no noroeste do estado de São Paulo. Neotropical Entomology 34: 475-484.

Demite, P. R. \& R. J. F. Feres. 2005. Influência de vegetação vizinha na distribuição de ácaros em seringal (Hevea brasiliensis Muell. Arg., Euphorbiaceae) em São José do Rio Preto, SP. Neotropical Entomology 34: 829-836.
Feres, R. J. F. 2000. Levantamento e observações naturalísticas da acarofauna (Acari, Arachnida) de seringueiras cultivadas (Hevea spp., Euphorbiaceae) no Brasil. Revista Brasileira de Zoologia 17: $157-173$

Feres, R. J. F.; D. de C. Rossa-Feres; R. D. Daud \& R. S. Santos. 2002. Diversidade de ácaros em seringueiras (Hevea brasiliensis Muell. Arg., Euphorbiaceae) na região noroeste do estado de São Paulo, Brasil. Revista Brasileira de Zoologia 19: 137-144.

Ferla, N. J. \& G. J. de Moraes. 2002. Ácaros (Arachnida, Acari) da seringueira (Hevea brasiliensis Muell. Arg.) no estado do Mato Grosso, Brasil. Revista Brasileira de Zoologia 19: 867-888.

Ferla, N. J. \& G. J. de Moraes. 2003a. Ciclo biológico de Calacarus heveae Feres, 1992 (Acari, Eriophyidae). Revista Brasileira de Entomologia 47: 399-402.

Ferla, N. J. \& G. J. de Moraes. 2003b. Efeito de diferentes concentrações de acaricidas e inseticidas-acaricidas sobre Calacarus heveae Feres, 1992 e Tenuipalpus heveae Baker, 1945 (Acari: Eriophyidae e Tenuipalpidae). Acta Biologica Leopoldensia 25: 179-185.

Flechtmann, C. H. W. 1975. Elementos de Acarologia. São Paulo, Livraria Nobel S.A., 344 p.

Gallo, D.; O. Nakano; S. Silveira-neto; R. P. L. Carvalho; G. C. Baptista; E. Berti-Filho; J. R. P. Parra; R. A. Zucchi; S. B. Alves; J. D. Vendramim; L. C. Marchini; J. R. S. Lopes \& C. Omoto. 2002. Entomologia agrícola. Piracicaba, FEALQ, 920 p.

Gonçalves, P. S.; O. C. Bataglia; A. A. Ortolani \& F. S. Fonseca. 2001. Manual de heveicultura para o estado de São Paulo. Boletim Técnico IAC 189, $77 \mathrm{p}$.

Hernandes, F. A. \& R. J. F. Feres. 2006. Diversidade e sazonalidade de ácaros (Acari) em seringal (Hevea brasiliensis Muell. Arg.) no noroeste do estado de São Paulo. Neotropical Entomology 35: $523-535$.

Instituto Agronômico de Campinas (IAC). 2006. Programa seringueira: Importância da cultura. Disponível em: http://www.iac.sp.gov.br. Acesso: 26 de junho de 2007.

Pontier, K. J. B.; G. J. de Moraes \& S. Kreiter. 2000. Biology of Tenuipalpus heveae (Acari, Tenuipalpidae) on rubber tree leaves. Acarologia 41: 423-427.

Tanzini, M. R. 1999. Manejo integrado do percevejo-de-renda da seringueira e ácaros na Hevea, p. 31-44. In: Gonçalves, P. S. \& J. F. C. Benesi. 1st. Ciclo de palestras sobre heveicultura paulista. Barretos, SP.

Vieira, M. R. \& E. C. Gomes. 1999. Sintomas, desfolhamento e controle de Calacarus heveae Feres, 1992 (Acari: Eriophyidae) em seringueira (Hevea brasiliensis Muell. Arg.). Cultura Agronômica 8: $53-71$.

Vieira, M. R. \& E. C. Gomes. 2001. Avaliação de acaricidas no controle de Calacarus heveae Feres, 1992 (Acari: Eriophyidae) em seringueira através de contagem em campo. Cultura Agronômica 10: $145-158$.

Vieira, M. R.; E. G. Fabri \& E. A. Oliveira. 2000. Sintomatologia do ataque de Calacarus heveae em seringueira (Hevea brasiliensis). Revista de Agricultura 75: 405-414.

Vis, M. J. de; G. J. de Moraes \& M. R. Bellini. 2006. Mites (Acari) of rubber trees (Hevea brasiliensis Muell. Arg., Euphorbiaceae) in Piracicaba, State of São Paulo, Brazil. Neotropical Entomology 35: $112-120$.

Zar, J. H. 1999. Biostatistical analysis. New Jersey, Prentice-Hall Inc., $4^{\mathrm{a}}$ ed., $663 \mathrm{p}+212 \mathrm{App}$. 